\title{
NEL MERCATO DEI NON SAGGI: LO SFRUTTAMENTO DELLA VITA IN DANTE (PRIMI ACCENNI PER UNA RICERCA)
}

\section{The Exploitation of Life in Dante}

\author{
Juan Varela-Portas de Orduña
}

Universidad Complutense de Madrid

\section{Sommario}

In questo lavoro si esplora la possibilità che l'inserzione dello sfruttamento umano all'interno del processo lavorativo e la conseguente apparizione sociale dell'uomo-merce abbiano lasciato tracce nell'opera di Dante. Si rintracciano così in una selezione di passi i problemi che questo processo impone alla nascente concezione dell'identità individuale e all'assunzione in una cornice ideologica ancora sacralizzata della distinzione fra ambito pubblico e ambito privato, che caratterizzerà il capitalismo fino a poche decadi fa.

\section{Parole chiave}

Dante; economia politica medievale; esilio; povertà; merce

\begin{abstract}
This article explores the possibility that the introduction of human exploitation within the labor process and the consequent appearance in society of the man-commodity may have left traces in Dante's works. Looking at selected passages, the essay recognizes the problems that such a process creates for the rising conception of individual identity and for the adoption - in a still sacralized ideological framework - of the distinction between the public and private domains that characterized capitalism until a few decades ago.
\end{abstract}

Keywords

Dante; medieval politic economy; exile; poverty; merchandise 
1. Nelle ultime decadi, lo studio dei materiali intellettuali, letterari e, più genericamente, culturali con i quali Dante costruisce le sue opere ha sperimentato un aumento in qualità e quantità veramente esponenziale, indotto probabilmente dall'eccezionale fioritura che i cosiddetti studi culturali hanno avuto nel mondo accademico. Gli studi su Dante hanno approffondito - e continuano ad approffondire - non solo le tradizionali questioni letterarie, politiche, filosofiche e teologiche che incidono sull'opera dantesca ma anche il rapporto di essa con la cultura popolare, l'omiletica, la predicazione, la devozione mariana, la letteratura francescana e tanti altri elementi culturali importantissimi nella società fra Duecento e Trecento.

Forse un po' meno si è indagato sulle esperienze psicosociali che, «interpellando» ideologicamente il soggetto, condizionano o piuttosto costruiscono l'uso, cosciente e incosciente, che Dante dà a quei materiali, forse perché tali esperienze sono molto meno accessibili visto che, appartenendo a quello che Lacan ha chiamato il Reale e che noi dantisti possiamo leggere come una causa assente (come Dio o la materia prima) che si percepisce solo attraverso i suoi «sintomi», devono essere necessariamente ipotizzate, il che contravviene l'atteggiamento diremmo «prudente», che anche negli ultimi tempi è norma diffusa nella dantistica. ${ }^{1}$ Tuttavia crediamo che cercare di indagare su come possano agire quelle cause assenti traumatiche, che si esprimono attraverso sintomi testuali, è importante per capire l'opera di Dante, appunto perché essa si produce in un momento storico di grande crisi e cambiamento ideologici, in cui i materiali ereditati, provenienti dal mondo feudale, si usano in una società, o, se si vuole, in un insieme di esperienze psicosociali, radicalmente diverso da quello in cui quei materiali erano stati creati; un mondo, per dirla con Gramsci, in cui le nozioni antiche non servono più per spiegare il mondo ma non sono ancora nate nozioni e materiali culturali nuovi più adatti al momento di profonda crisi e cambiamento sociale in cui scrive Dante. E non dobbiamo dimenticare che la letteratura è innanzi tutto un modo di fare cose con parole, che il suo scopo è quello di produrre effetti psicosociali nel mondo, come si indica molto chiaramente a proposito della Commedia nell'Epistola a

\footnotetext{
${ }^{1}$ Sul concetto di inconscio ideologico, di radice althusseriana e freudiana, creato da Juan Carlos Rodríguez, vd. Rodríguez, J.C., «La literatura y la pesadilla del yo. Freud y los dos inconscientes», in A. Conde et al. (a c. di), Matrices del siglo xx: signos precursores de la Modernidad, Madrid, Universidad Complutense de Madrid, 2001, pp. 393-413; Id., «El yo libre en el inconsciente del neoliberalismo actual», Pensar desde abajo, 1 (2012), http://pensardesdeabajo.org/articulos/el-yo-libre-en-elinconsciente-del-neoliberalismo-/; Id., «Sobre el inconsciente ideológico y la radical historicidad de la literatura», in Id., De qué hablamos cuando hablamos de marxismo, Madrid, Akal, 2013, pp. 69-109; Althusser, L., Positions, París, Editions sociales, 1976; Becerra Mayor, D., La novela de la no ideología, Cádiz, Tierra de nadie, 2013, pp. 9-41; Muzzioli, F., L'alternativa letteraria, Roma, Meltemi, 2001, pp. 101-123; Giordano, Ch., «Di cosa parliamo quando parliamo di ideologia: un'approssimazione al concetto d'inconscio ideologico di Juan Carlos Rodríguez», in S. Albertazzi, F. Bertoni, E. Piga, L. Raimondi e G. Tinelli (a c. di), L'immaginario politico. Impegno, resistenza, ideologia, Between, v. 10 (2015), http://www.Betweenjournal.it/; Ead., «La teoría del inconsciente ideológico de Juan Carlos Rodríguez: una línea de fuga entre marxismo, psicoanálisis y estudios literarios», Romanica Olomucensia, 30/1 (2018), pp. 111-124.
} 
Cangrande, ${ }^{2}$ e che perciò non possiamo trascurare il fatto che l'opera di Dante si inserisce in un mondo economico e sociale in profonda trasformazione, così come gli effetti che in essa produce, appunto, quella trasformazione in corso.

In questo senso, riteniamo che nelle ultime decadi si è forse dimenticato il fatto fondamentale che l'opera di Dante nasce contemporaneamente alle prime esperienze psicosociali di tipo capitalista nelle città italiane. Non entreremo ora nella vexata quaestio fra storici, purtroppo non fra dantisti - delle origini del capitalismo, cioè nelle discussioni fra quelli che le identificano con la diffusione, già nel dodicesimo secolo, dei rapporti mercantili e della finanziarizzazione delle tasse e gabelle feudali, come fa ad esempio Pirenne, e quelli invece che parlano di capitalismo soltanto quando si forma un'organizzazione sociale o un modo di produzione dominante, cioè con rapporti sociali e politici strutturati e manifesti, come fa ad esempio Romano. ${ }^{3}$ A nostro parere, al di là della questione, non si può dubitare che nei comuni toscani della seconda metà del Duecento ci siano già quelle che potremmo chiamare esperienze psicosociali capitalistiche in modo sufficientemente diffuso tali da produrre «sintomi» nelle soggettività e pertanto nei testi letterari, ${ }^{4} \mathrm{o}$, per dirla più tecnicamente, per attuare «sintomaticamente» - non come causaeffeto ma come trauma-sintomo, cioè per condensazione e spostamento - sull'ambito ideologico-culturale. ${ }^{5}$ Ora, fra queste esperienze, ce ne sono tre assolutamente fondamentali

${ }^{2}$ «Finis totius et partis esse posset multiplex, scilicet propinquus et remotus; sed, omissa subtili investigatione, dicendum est breviter quod finis totius et partis est removere viventes in hac vita de statu miserie et perducere ad statum felicitatis» (Epistola XIII 15; citiamo da Alighieri, D., Epistole, a c. di C. Villa, in Id., Opere, edizione diretta da M. Santagata, Milano, Mondadori, 2014, vol. 2, p. 1506). Claudia Villa, nel suo commento al passo citato (p. 1589), stabilisce un parallelismo tra la funzione dell'opera come in esso si delinea e la funzione di pontefice e imperatore nel finale della Monarchia, il che sottolinea la funzione «psicosociale» dell'opera. Ma si veda anche l'inizio del Convivio, in cui questa finalità è altrettanto palese.

${ }^{3}$ Vd. Pirenne, H., Storia economica e sociale del Medioevo, Milano, Garzanti, 1967; Romano, R., Tra due crisi: l'Italia del Rinascimento, Torino, Einaudi, 1971. A questo proposito, continua ad essere imprescindibile il dibattito tenuto fra Dobb, Hilton, Procacci, Lefebvre, Hill e Takahashi raccolto nel volume Bolaffi, G. (a cura di), La transizione dal feudalesimo al capitalismo, Roma, Savelli, 1974 [edizione spagnola: Dobb, Maurice-Hilton, Rodney et al., La transición del feudalismo al capitalismo, Barcelona, Crítica, 1982], così come, naturalmente, le illuminanti pagine dedicate da Marx al riguardo nel quaderno VI dei Grundrisse (vid. Marx, K., Forme economiche precapitalistiche, prefazione di E.J. Hobsbawn, Roma, Editori Riuniti, 1970). Anche Jones, Ph., Economia e società nell'Italia Medievale, Torino, Einaudi, 1980.

${ }^{4}$ Oltre, evidentemente, a conseguenze prettamente sociali, che riassume perfettamente Simonetta Teucci: «Per dirla con Marx, il fiorino era la carica che dissolveva i legami che avevano tenuto salda la comunità entro la cerchia antica. Infatti Marx, sostiene che il denaro è la comunità, e che non può tollerarne un'altra che gli sia 'superiore'; in questo caso la comunità è quella della città comunale». (Teucci, S., «Il Medioevo», in Ead., L'infinito potere del denaro. Eredità, debiti, guadagni tra realtà e letteratura, Roma, Aracne, 2019, p. 56).

${ }^{5}$ La descrizione per noi non è quindi quella del marxismo classico in cui la cosiddetta «struttura» si manifesta o sovradetermina la cosiddetta «sovrastruttura» in un rapporto deterministico di causaeffetto. Partiamo invece da uno schema triadico in cui l'ambito socio-economico, l'ambito ideologico- 
che, a nostro parere, costituiscono il midollo, il seme del modo di produzione capitalista, e che nel momento in cui Dante scrive sono già largamente sviluppate nella società comunale: la conversione del denaro in capitale, l'apparizione della forma-merce e quella che riteniamo la causa di queste due, nonché elemento primordiale del capitalismo, e cioè lo sfruttamento all'interno del processo lavorativo. A nostro parere queste dinamiche sono già così estese nel mondo di Dante che lasciano tracce sintomatiche nelle sue opere: ad esempio, l'apparizione della forma-merce - che non si deve confondere con la semplice merce materiale - e del capitale - cioè del denaro padrone di sé stesso e padrone degli uomini - si manifesta sintomaticamente in alcuni passi molto significativi di Le dolce rime, Doglia mi reca e il Convivio, attraverso la descrizione dei suoi effetti metafisici e psicologici. ${ }^{6}$ Vorremmo ora trattare, in forma ancora iniziale, ${ }^{7}$ qualcosa che ci sembra ancora più difficile, e cioè rintracciare in che modo lo sfruttamento all'interno del processo di produzione-lavoro può condizionare o costruire o interpellare ideologicamente Dante e la sua opera.

Lo sfruttamento all'interno del processo lavorativo è la grande e terribile creazione sociale del capitalismo e si produce in maniera socialmente effettiva con la manifattura dispersa del tessile nella seconda metà del Duecento nelle città toscane, sicuramente a Siena

culturale e l'ambito politico-giuridico (che, mutatis mutandis, coincidirebbero rispettivamente con il Reale, l'Immaginario e il Simbolico lacaniani), intrecciati come un nodo borromeo, stabilirebbero rapporti mutui in cui comunque l'ambito socio-economico agisce dall'«indietro», dall'«ombra», come «causa assente», conformando e deformando per metafora e metonimia, sugli altri due ambiti. A sua volta l'ambito ideologico-culturale, ovvero l'inconscio ideologico, agisce per condensazione e spostamento sull'ambito politico-giuridico e quindi sul testo «cosciente» dell'opera artistica.

${ }^{6}$ Questo è l'argomento di un altro lavoro, ancora in processo di elaborazione, complementare a questo che qui presentiamo, in cui cercheremo di argomentare che appunto l'apparizione della formamerce e del denaro-capitale sarà percepita da Dante come perversione della forma sostanziale e quindi assimilata alla materia informe che vi sottostà. Ma sul tema della conversione del denaro in capitale alla fine del Medioevo, si veda almeno: Le Goff, J., Lo sterco del diavolo. Il denaro nel Medioevo, Bari, Laterza, 2012; Id., La Edad Media y el dinero: ensayo de antropología histórica, Madrid, Akal, 2012; Todeschini, G., I mercanti il tempio. La società cristiana eil circolo virtuoso della ricchezza fra Medioevo ed Età Moderna, Bologna, Il Mulino, 2002; Capitani, O. (a c. di), L'etica economica medievale, Bologna, Il mulino, 1974. Più puntati sulla letteratura e in particolare su Dante: Klettke, C., «Il vaglio di beni terrestri e celesti. Etica economica ed 'economia dell'anima'», in A. Barbieri e E. Gregori (a c. di), Letteratura e denaro. Ideologie metafore rappresentazioni. Atti del XLI Convegno Interuniversitario (Bressanone, 11-14 luglio 2013), Padova, Esedra, 2014, pp. 155-189; Day, W.R. Jr., «Economy», in Baransky, Z. G. and Pertile, L. (a c. di), Dante in Context, Cambridge, Cambridge University Press, 2015, pp. 30-46; Montefusco, A., «Banca e poesia al tempo di Dante», in Quaderno 58, Milano, Associazione per lo sviluppo degli studi di banca e borsa dell'Università del Sacro Cuore, 2017, pp. 9-45; e vorremmo segnalare specialmente, per le chiavi di lettura di cui ci forniscono, Teucci, S., «Il Medioevo», op. cit., pp. 35-67; e Fenzi, E., «Tra etica del dono e accumulazione. Note di lettura alla canzone dantesca Doglia mi reca», in Grupo Tenzone, Doglia mi reca ne lo core ardire, Umberto Carpi (a c. di), Madrid, Departamento de Filología Italiana UCM - Asociación Complutense de Dantología, 2008, pp. 147-211.

${ }^{7}$ Questo lavoro, come il sottotitolo cerca di indicare, si pone molto modestamente come punto di partenza di una ricerca che speriamo portar avanti nei prossimi tempi. 
e Firenze. ${ }^{8}$ Le sue conseguenze sulla soggettività umana sono decisive: nel feudalesimo, lo sfruttamento si produceva dopo il momento della produzione sotto forma di sottrazione in base a 'diritti' fondati ideologicamente e politicamente, ma il contadino o il servo controllavano il processo di produzione e ne erano 'padroni'. Con la produzione capitalistica l'operaio di una bottega associata a una manifattura dispersa non è più padrone del processo produttivo, non controlla la sua funzione, le sue ragioni e obiettivi e soprattutto il suo esito economico, ma semplicemente apporta al processo la sua vita personale - quello che secoli dopo i teorici liberali chiameranno «forza lavoro» - sotto forma di tempo, di sforzo fisico corporale e di conoscenze normalmente tramandate dalla tradizione e delle quali ora il vero padrone si impossessa in forma di plusvalore. La chiave, come si sa, è che quello che esce dal processo produttivo non è più una cosa - una natura - ma una merce con un plusvalore del quale si impossessa la persona o le persone che controllano il processo nel suo insieme e che retribuiscono con un salario una parte della vita che gli operai apportano al processo. La conseguenza è, come dicevamo, terribile perché in questo modo la persona diventa formamerce e deve vendersi come tale in un mercato in cui il potere ormai non proviene da diritti ideologici o politici ma dal controllo della proprietà e cioè del processo lavorativo.

Ora, la conversione dell'essere umano in forma-merce produce due complessissimi processi ideologici incoscienti, che sono quelli che ripercorreranno la cosiddetta «modernità» nel suo insieme (intesa sia come «età moderna», come fanno gli storici, sia come il periodo del capitalismo industriale, come si fa piuttosto in ambito filosofico): il primo e basilare è l'identificazione dell'identità con un'individualità che uno deve necessariamente vendere nel processo lavorativo; il secondo è la dissociazione dell'identità individuale in un versante privato - quello che, almeno fino al periodo postmoderno, non si mette in vendita, cioè l'intimità, l'anima, la coscienza, il rapporto con Dio, ecc. - e un versante pubblico che si mette in vendita ed è perciò pervaso di nozioni come «rappresentazione», «immagine», «comunicazione», «scambio», ecc. Crediamo che in Dante questa distinzione fra l'io privato e l'io pubblico è già molto percepibile, e che in essa si avverte il dramma dell'uomo che in uno stato capitalista deve necessariamente vendersi nel mercato dei non saggi.

2. La prima parte della canzone Poscia ch'Amor, quella più satirica e critica contro i falsi leggiadri, è, a nostro parere, costruita in base a questa costruzione ideologica ormai pienamente capitalistica. ${ }^{9}$ Come si sa, in questa prima parte, la canzone è una guida per

${ }^{8}$ Al riguardo si vedano Rutenburg, V., Popolo e movimenti popolari nell'Italia del '300 e '400, Bologna, Il Mulino, 1971; e Franceschi, F., «Il mondo della produzione urbana. Artigiani, salariati, Corporazioni», in F. Franceschi (a c. di), Storia del lavoro in Italia, Il Medioevo. Dalla dipendenza personale al lavoro contrattato, Roma, Castelvecchi, 2017, pp. 374-420.

${ }^{9}$ «Si en Le dolci rime Dante busca construir una nueva noción de identidad privada (que tenga, claro está, su reflejo en el mundo público), en Poscia ch'Amor busca construir las bases de una nueva legitimidad pública social. Asistimos, pues, al trabajoso nacimiento (a la trabajosa construcción) de la distinción ideológica entre lo público y lo privado - necesaria para (y causada por) la consolidación de las primeras relaciones sociales capitalistas -, y, con ello, a uno de los primeros 
interpretare i segni o manifestazioni visibili della leggiadria, che dovrebbe trovarsi sempre unita alla virtù, cioè alla nobiltà dell'anima. Invece, i segni sociali della leggiadria possono essere ingannevoli e ci possiamo quindi trovare davanti a «falsi cavalier', malvagi e rei» (v. 112) che ostentano apparenza leggiadra e sono considerati leggiadri. Abbiamo chiamato questa canzone «la canzone dell'apparenza» perché in questa prima parte cerca di mettere in rilievo l'inganno su cui si sostengono le apparenze sociali. ${ }^{10}$ In essa, l'ambito collettivo è denunciato come l'ambito dell'inganno, della falsità, dell'apparenza sensibile: i falsi leggiadri provocano «l'inganno / di loro e della gente / c'hanno falso giudicio in lor sentenza» (vv. 29-31), sono sempre preoccupati di essere giudicati da persone facili da ingannare (vv. 39-44) e si accontentano di essere ammirati dal vulgo (vv. 46-47). Ora, i versi che seguono mostrano chiaramente che questo atteggiamento è un modo di vendersi, cioè di considerare la propria vita in rapporto al valore mercantile - e non si trascuri qua il ruolo fondamentale della nozione di «valore» e la sua trasformazione in questo periodo- ${ }^{11}$ che deriva direttamente dall'immagine pubblica che si trasmette agli altri.

Sono che per gittar via loro avere

credon potere

esfuerzos por definir el ámbito de lo social como independiente del ámbito de 'lo natural' (aunque. lógicamente, no plenamente exitoso)». (Varela-Portas de Orduña, J., «Presentación a Poscia ch'Amor del tutto m'ha lasciato", in Dante Alighieri, Libro de las canciones y otros poemas, a c. di J. Varela-Portas de Orduña (coord.), R. Arqués Corminas, R. Pinto, R. Scrimieri Martín, E. Vilella Morató y A. Zembrino, traducción de R. Pinto, Madrid, Akal, 2014, p. 305). Per l'analisi e l'interpretazione della canzone rimandiamo al nostro commento (pp. 304-331) e bibliografia pregressa (a p. 307), ma anche a Varela-Portas de Orduña, J., «Doctrina y política en las canciones político-doctrinales de Dante», Tenzone, 15 (2014), pp. 139-182, specialmente pp. 152-161.

${ }^{10}$ «Si las virtudes morales son signos, siempre inconfundibles, de la nobleza, la leggiadria, para ser tal, debe ser signo de esas virtudes, y aunque en sí misma no sea virtud (vv. 77-88), solo es verdadera si está ligada a ellas. Si no es así, no podemos hablar de leggiadria, sino de falsas apariencias, de comportamientos sociales engañosos que no están sostenidos por un contenido de verdad. En este sentido, podríamos denominar a esta canción, al menos en toda su primera parte (vv. 20-57), la más directamente político-social, la menos doctrinal y más satírica y de denuncia, la 'canción de la apariencia', porque trata de poner de manifiesto el engaño en que se sostienen ciertas apariencias sociales. En ella el ámbito de lo colectivo es denunciado repetidamente como el ámbito del engaño, la falsedad y la apariencia, que hace que lo que debería ser bello y virtuoso sea en verdad grosero y vil (v. 10)». (Varela-Portas de Orduña, «Doctrina y política en las canciones políticodoctrinales de Dante», op. cit., pp. 153-154).

${ }^{11}$ Una delle spie principali dei profondi cambiamenti socio-economici e ideologici svoltisi in questo periodo è la mutazione di senso delle nozioni di «virtù» e «valore», le quali, attraverso un processo complesso, ancora da indagare in profondità, passano dall'indicare, rispettivamente, la capacità di influsso ontologico sugli esseri che sottostanno nella gerarchia universale e il posto che in questa gerarchia è assegnato grazie appunto alla virtù ricevuta, al significare la capacità di intervento civile nella comunità e il prestigio che in essa si detiene, cosicché la nozione di «valore» passa ad avere un rapporto diretto con la posizione e la funzione all'interno del processo produttivo. 
capere là dove li boni stanno

che dopo morte fanno

riparo nella mente

a quei cotanti c'hanno conoscenza.

Ma lor messione a' bon' non può piacere,

perché tenere

savere fôra, e fuggirieno il danno

che s'agiugne a lo 'nganno

di loro e della gente

c'hanno falso giudicio in lor sentenza.

Qual non dirà fallenza

divorar cibo ed a lussuria intendere,

ornarsi come vendere

si dovesse al mercato d'i non saggi?

ché 'l saggio non pregia om per vestimenta,

ch'altrui sono ornamenta,

ma pregia il senno e li gentil coraggi.

Poscia ch'Amor 20-38 (corsivo nostro)

A nostro parere, Dante in questo passo dipinge la figura dello scialacquatore non tanto come vizioso di vino e taverna, come vuole De Robertis, ma come la persona che fa ostentazione della sua ricchezza per guadagnare un prestigio o, come diremmo oggi, un capitale sociale o politico, caratteristica propria della nuova classe mercantile. ${ }^{12}$ Lo scialacquatore, con il suo sperpero (la «messione» del v. 26), cerca di ingannare «a quei cotanti c'hanno conoscenza» (v. 25) ma in realtà ci riesce soltanto con «la gente / c'hanno falso giudicio in lor sentenza» (v. 31). La smisurata spesa serve a produrre un'immagine sociale, pubblica, tutta sensitiva, e quindi un'apparenza di «valore» nello scambio sociale che si concepisce come scambio mercantile. Dante presenta in questi versi la società come un grande mercato di sciocchi, cioè di persone «c'hanno falso giudicio in lor sentenza», in cui il falso leggiadro si mette in vendita in virtù della sua apparenza fisica, caratterizzata appunto dal modo di vestire, che non è più una forma di indicare il luogo naturale assegnato alla persona dalla provvidenza, come nel puro feudalesimo, ma un ornato che proietta una falsa apparenza che non corrisponde più all'essere sociale-naturale (vv. 3637) - il che, tra l'altro, spiega le cause ideologiche della grande fioritura dell'industria tessile nella società comunale e signorile, conseguenza della necessità che sentiva il magnate mercantile di mostrare nobiltà e rango sociale. ${ }^{13}$ Risulta molto interessante il

${ }^{12}$ Per un dibattito sul significato e senso del passo, rimandiamo al nostro commento ad locum, in Dante Alighieri, Libro de las canciones, op. cit., p. 314.

${ }^{13}$ Prima dei grandi cambiamenti sociali del Duecento, l'abbigliamento denotava con chiarezza il luogo «naturale» della persona, ed era perciò inteso come una apparenza somigliante della traccia divina impressa nel sangue, ma con l'estensione dei rapporti mercantili l'abbigliamento non può più garantire il ruolo «naturale» della persona e diventa quindi una apparenza dissomigliante, come 
dubbio che esprime Pernicone nell'edizione Barbi-Pernicone: «Sarà da interpretare 'al mercato in cui si mettono in vendita i non saggi', oppure 'al mercato in cui a giudicare per comprare sono persone non sagge'?».14 La risposta è, chiaramente, entrambe le interpretazioni. Dante percepisce il mondo mercantile come un mondo in cui la ragione, che dovrebbe vedere la verità nascosta sotto le apparenze, viene a meno, e l'uomo diventa semplice rappresentazione, immagine pubblica, merce in vendita.

3. È molto interessante che Dante sia stato capace di percepire questa nuova condizione umana già nel periodo fiorentino, quando probabilmente la sua soggettività non si è ancora sentita direttamente interpellata da essa, essendo lui un rentier - non ricchissimo ma in qualche modo certamente agiato - che vive delle rendite probabilmente frutto dell'usura del padre e del nonno. ${ }^{15}$ Ma la situazione cambia radicalmente nell'inverno del 1302 quando, all'improvviso, dopo il bando che lo manda in esilio, per sopravvivere, Dante dovrà necessariamente vendersi come merce nel mercato dei non saggi, offrire al miglior offerente la sua stessa vita individuale, cioè il suo tempo, il suo sforzo fisico e mentale e le sue conoscenze e abilità personali. Come indica Sabrina Ferrara:

dimostrano, ad esempio, lo svestirsi in piazza di Francesco di Assisi nel momento della sua crisi o conversione, o la narrazione di Cacciaguida sul modo di vestire degli antichi fiorentini nel XV del Paradiso (Par. XV 100-102, 112-117). In questa situazione, l'inconscio ideologico feudale, secondo il quale mostrare nobiltà implica essere nobile, si mescola con l'inconscio ideologico ormai capitalista per il quale l'immagine pubblica indica il valore come merce della persona.

${ }^{14}$ Pernicone, V., ad locum, in Dante Alighieri, Rime della maturità e dell'esilio, a cura di M. Barbi e V. Pernicone, Firenze, Le Monnier, 1969, p. 443.

15 «Le attività degli Alighieri - i quali si dedicavano per tradizione al commercio del denaro, come attestano i documenti sopravvissuti - avevano indubbiamente garantito alla famiglia una certa agiatezza [negli anni ' 40 del Duecento]» ma «Sicuramente mancò loro un patrimonio fondiario ampio, tale da garantire uno status distinto in una fase economica fortemente progressiva: gli unici contratti legati alla terra nei quali sono coinvolti sembrano più funzionali alla loro attività di prestatori che a una gestione consolidata del patrimonio» (Faini, E., «Ruolo sociale e memoria degli Alighieri prima di Dante», Reti Medievali, 15/2 (2014), Dante attraverso $i$ documenti I. Famiglia e patrimonio (secolo XII-1300 circa), a c. di G. Milani e A. Montefusco, p. 222 e p. 236). Elisa Brilli puntualizza: «l'attività politica [di Dante] non migliorò certamente le condizioni economiche della famiglia a giudicare dal susseguirsi dei mutui contratti prima da Dante e dal fratello Francesco con terzi (di 277 fiorini d'oro l'11 aprile 1297, di 480 fiorini d'oro il 23 dicembre seguente, di 53 fiorini d'oro il 23 ottobre 1299) e poi da Dante con Francesco, le cui condizioni dovevano essersi risollevate (di 125 fiorini d'oro il 14 marzo 1300 e poi, s'ignora se in accumulo o in estinzione, di 90 fiorini l'11 giugno 1300)» (Brilli, E., «Dante Alighieri. Biografia e bibliografia», in C. Ossola (a c. di), Dante, Roma, Istituto della Enciclopedia Italiana Treccani, 2011, pp. LXXXVIII-LXXXIX). Ma sulla questione delle basi economiche del Dante fiorentino si veda tutto il numero citato (15, 2 [2014]) di Reti medievali (http://rivista.retimedievali.it). E per ulteriori approfondimenti, la recente edizione del Codice Diplomatico Dantesco: Dante Alighieri, Le opere, vol. VII Opere di dubbia attribuzione e altri documenti danteschi, tomo III Codice diplonatico dantesco, a c. di T. De Robertis, G. Milani, L. Regnicoli e St. Zamponi, Roma, Salerno, 2016. 
La perdita di ogni bene materiale, di ogni essenza sociale, di ogni appartenenza civica pone Dante nella condizione di elaborare una nuova rinascita e di ridare contenuto a una credibilità che gli era stata tolta con il bando; ed è questa obbligatorietà ad autoricostruirsi, a tutti i livelli di vita, che determina il suo percorso e la modelizzazione progressiva della propria immagine. ${ }^{16}$

A nostro parere, la questione di base che non dobbiamo dare per scontato è il fatto che la condizione economica di Dante cambia radicalmente, passando da essere un rentier più o meno agiato, ma comunque sia non precario, ad essere quello che potremmo chiamare un freelance in uno stato assoluto di precarietà - in tempi in cui la precarietà significava rischio immediato di morte - che deve necessariamente vendere i suoi servizi nelle decadenti corti feudali italiane - senza neanche avere un profilo «professionale» adatto a tale compito - e quindi sentire sulla propria carne lo sfruttamento della vita individuale consustanziale al modo di produzione capitalista. ${ }^{17}$ È vero che, come si sa, i numerosissimi esiliati comunali avevano intessuto tra di loro reti di solidarietà e aiuto collettivo, ${ }^{18}$ ma è anche vero che l'esilio di Dante ha delle caratteristiche personali e politiche che lo rendono particolarmente crudele, soprattuttto dopo la rottura dei legami con i compagni della parte bianca nel 1304 . Come spiega Elisa Brilli:

Così, si potrebbe argomentare che, se il bando di Dante non fu singolare come provvedimento in sé, lo diventò nei fatti, e tanto più dopo lo sfaldamento dell'Universitas alborum (1304), perché la condizione di bandito veniva a combinarsi, da un lato, con una famiglia di risorse economiche non ingenti e di visibilità limitata intra e extra moenia; dall'altro, con una professionalità che, senza nulla togliere a «qual che si fosse, il suo ingegno», mal si accordava con il mercato professionale d'inizio Trecento, consentendogli di fungere al più da mediatore diplomatico al soldo di potentati locali, si pensi al servizio prestato ai Malaspina presso il vescovo di Luni. Insomma,

\footnotetext{
${ }^{16}$ Ferrara, S., La parola dell'esilio. Autori e lettori nelle opere di Dante in esilio, Firenze, Franco Cesati, 2016, p. 15.

${ }^{17}$ È vero che, come indica Franziska Meier, non è facile «to understand what the courts at that time looked like and what role an exiled poet could have figured out to play in them [...]. Dante`s self-fashioning in the Convivio as a solemn educator of courtiers could only have amounted to a totally inappropriate attempt, a self-conceit or even an involuntary joke». (Meier, F., «Introduction», in Dante's Convivio. Or How to Restart a Career in Exile, a c. di F. Meier, Oxford-Bern-Berlin-Bruxelles-New York-Wien, Peter Lang, 2018, p. 5). Comunque, al di là delle difficoltà per capire la portata e il successo o fallimento dell'operazione di auto-promozione proiettata, ma non portata avanti - non si deve mai dimenticare questo aspetto - da Dante nei primi anni dell'esilio, la questione importante, a nostro parere, è appunto che Dante sentisse quella estrema necessità di self-fashioning che, secondo noi, è indizio del suo bisogno materiale di vendersi in un mercato di non saggi.

${ }^{18}$ Ricciardelli, F., «Exile as evidence of civic identity in Florence in the time of Dante: Some examples», Reti Medievali, 5/1 (2004), pp. 1-15; Id., The politics of exclusion in Early Renaissance Florence, Turnhout, Brepols, 2007; Milani, G., L'esclusione dal Comune. Conflitti e bandi politici a Bologna e in altre città italiane tra XII e XIV secolo, Roma, ISIME, 2003.
} 
quell'eccentricità sociale ed economica che faceva spiccare il brillante trentenne nella cerchia di Cavalcanti così come il suo cursus di studi opaco, certamente non concluso, e l'iscrizione solo formale (e strumentale) all'Arte dei Medici e degli Speziali resero forse, girata la ruota e una volta fuori Firenze, la sua condizione di esule sensibilmente diversa da quella sperimentata da altri della medesima generazione, i quali potevano invece contare su risorse familiari, reti di contatti e competenze professionali più solide di quelli di Dante. ${ }^{19}$

Il fatto è che, caduto all'improvviso nella misera condizione di esule, Dante si trova di fronte, inaspettatamente, alla povertà avvertita come attributo della propria condizione individuale, avvertita, cioè, come paura e insicurezza ma soprattutto come stigma personale:

Poi che fu piacere delli cittadini della bellissima e famosissima figlia di Roma, Fiorenza, di gittarmi fuori del suo dolce seno - nel quale nato e nutrito fui in fino al colmo della vita mia, e nel quale, con buona pace di quella, desidero con tutto lo core di riposare l'animo stancato e terminare lo tempo che m'è dato -, per le parti quasi tutte alle quali questa lingua si stende, peregrino, quasi mendicando, sono andato, mostrando contra mia voglia la piaga della fortuna, che suole ingiustamente al piagato molte volte essere imputata. Veramente io sono stato legno sanza vela e sanza governo, portato a diversi porti e foci e liti dal vento secco che vapora la dolorosa povertade; e sono apparito alli occhi a molti che forse che per alcuna fama in altra forma m'aveano imaginato: nel conspetto de' quali non solamente mia persona invilio, ma di minor pregio si fece ogni opera, sì già fatta come quella che fosse a fare. (Convivio I III 4-5; corsivo nostro) ${ }^{20}$

Il passo, famosissimo, permette di capire che in quel momento difficile Dante sente la povertà, coscientemente o incoscientemente, non solo come conseguenza di una causa esterna e incontrollabile come la fortuna, ${ }^{21}$ ma anche, e forse soprattutto, come una condizione personale che condiziona fortemente la sua rappresentazione sociale e quindi la sua capacità di vendersi nel mercato professionale al quale ora aveva bisogno di accedere, in una dinamica della miseria come elemento di esclusione sociale dinamica non propriamente medievale - che si protrae fino ai nostri tempi. A questo

\footnotetext{
${ }^{19}$ Brilli, E., «L'arte di dire l'esilio», Bolletino di italianistica. La letteratura italiana el'esilio, 8/2 (2011), p. 20.

${ }^{20}$ Citiamo dall'edizione Fioravanti (Dante Alighieri, Convivio, a c. di G. Fioravanti, in Id., Opere, edizione diretta da M. Santagata, volume secondo, pp. 116-118).

${ }^{21}$ Come spiega Fioravanti nel suo commento (p. 119), «Il termine 'fortuna' ha per Dante significati più complessi che per noi [...] reggeva a suo capriccio il mondo delle ricchezze, del potere e del successo [...] era diventata una ancella di Dio [...]. Il suo girare [...] fa parte esso stesso di un ordine provvidenziale che il più delle volte sfugge alla nostra comprensione immediata». Sono molto celebri i versi che la definiscono in If. VII 77-84: «Similemente a li splendor mondani / ordinò general ministra e duce / che permutasse a tempo li ben vani / di gente in gente e d'uno in altro sangue, / oltre la difension d'i senni umani; / per ch'una gente impera e l'altra langue, / seguendo lo giudicio di costei, / che è occulto come in erba l'angue.»
} 
punto, si deve considerare il profondo, lungo e contraddittorio processo ideologico di desacralizzazione della povertà che si svolge dalla fine del Duecento fino a tutto il Trecento. In esso, con l'avvio delle prime pratiche sociali capitalistiche, la povertà passa da essere considerata un elemento naturale dell'ordine universale ad essere associata, con profonda angoscia, alla prima esperienza dell'uomo-merce, non solo da Dante ma anche da autori come Monte Andrea o, in un senso molto vicino al nostro autore, da un altro esiliato come Fazio degli Uberti. ${ }^{22}$ Dante vive la povertà, come tanti altri suoi contemporanei, come «la piaga della fortuna», ma allo stesso tempo come uno stigma che colpevolizza il povero, o impoverito, della sua stessa situazione, il che incide direttamente sul suo «valore» nel mondo e ostacola la percezione collettiva della sua natura nobile. In questo modo, la catena consequenziale che Dante stabilisce dal capitolo XVI al XXII del quarto trattato del Convivio, - dove appunto, di fronte al «valore» che producono le ricchezze, stabilisce il «valore / per lo qual veramente omo è gentile» (Le dolci rime 12-13) ${ }^{23}$ - cioè:

$$
\text { Dio - nobiltà di anima - abito eligente - virtù morali - felicità - elogio pubblico, }
$$

sarà spezzata a causa della povertà, che impedisce che le virtù della persona povera producano felicità ed elogio pubblico. Se la povertà fa sì che l'elogio o il vituperio pubblico si stacchino dal vero valore individuale - prodotto dalla semina della nobiltà da parte di Dio nell'anima individuale e dalla sua coltura da parte dell'individuo -, questo vuol dire che fra l'ambito privato, che segue le regole ontologiche del mondo naturale, e l'ambito pubblico si produce uno stacco, una breccia, che non risponde più alla dialettica somiglianza-dissomiglianza e alla dialettica virtù-valore proprie della concezione sacralizzata feudale. Così come le ricchezze - percepite ormai come forma merce - e il denaro - diventato capitale - implicano la rottura metafisica della forma sostanziale e cioè della natura, la povertà implica una rottura ontologica che distrugge la cascata di cause seconde che configura il mondo. In questo modo, la povertà resta saldamente vincolata al valore sociale individuale vissuto ormai non come una condizione ontologica ma appunto come rappresentazione e cioè come

\footnotetext{
${ }^{22}$ Sulla desacralizzazione della povertà alla fine del Medioevo, si vedano: Mollat, M., «Il concetto della povertà del Medioevo: problematica», in Capitani, O. (a c. di), La concezione della povertà nel Medioevo, Bologna, Patròn, 1983, pp. 1-34 [«La notion di pauvreté au Moyen Age: positions des problèmes», Revue d'Histoire de l'Eglise de France, t. LII, no 149 (1966), pp. 5-23]; Lis, C., Soly, H., Pobreza y capitalismo en la Europa preindustrial (1350-1850), Madrid, Akal, 1984 (specialmente i primi due capitoli); Manselli, R., «Da Dante a Coluccio Salutati. Discussions sur la pauvreté a Florence au XIV siècle», in Études su l'histoire de la pauvreté (Moyen Age-XVIe siècle), sous la direction de M. Mollat, Paris, Publications de la Sorbonne, 1974, vol. 2, pp. 637-659; La Roncière, C.-M. de, «Pauvres et pauvreté a Florence aun XIV siècle», in M. Mollat (dir.), Études su l'histoire de la pauvreté (Moyen Age-XVI siècle), Paris, Publications de la Sorbonne, 1974, vol. 2, pp. 661-745.

${ }^{23} \mathrm{Vd}$. Ardizzone, M.L., «Dante's 'Quaestio' on Nobility and the Criticism of Materialism», in F. Meier (a c. di), Dante's Convivio. Or How to Restart a Career in Exile, Oxford-Bern-Berlin-Bruxelles-New York-Wien, Peter Lang, 2018, pp. 156-157.
} 
vita-merce, in una dinamica che sarà ormai comune a tutta la vita capitalistica posteriore. ${ }^{24}$

Questo permette che emergano nei testi «sintomi» sociali della povertà, come succede nell'Epistola II, nella quale si vede come la povertà impedisce a Dante di assistere alle esequie del Conte Alessandro di Romena:

Doleat ergo, doleat progenies maxima Tuscanorum, que tanto viro fulgebat, et doleant omnes amici eius et subditi, quorum spem mors crudeliter verberavit; inter quos ultimos me miserum dolere oportet, quia a patria pulsus et exul inmeritus infortunia mea rependens continuo, cara spe memet consolabar in illo.

[...]

Ergo autem, preter hec, me vestrum vestre discretioni excuso de absentia lacrimosis exequiis; quia nec negligentia neve ingratitudo me tenuit, sed inopina paupertas quam fecit exilium. Hec etiam, velut effera persecutrix, equis armisque vacantem iam sue captivitatis me destrusit in antrum, et nitentem cunctis exsurgere viribus, hucusque prevalens, impia retinere molitur. (Epistola II 1; 3; corsivo nostro) ${ }^{25}$

Il passo è interessantissimo perché in esso si raccolgono tutte le perturbazioni ideologiche che stiamo analizzando: da una parte, è un'esplicita dichiarazione dello stato di dipendenza economica di Dante riguardo al signore di Romena, nel quale aveva riposto la sua speranza; dall'altra, vi si esprime, unita alla famosissima espressione di «exul

${ }^{24}$ Come sempre nel mondo medievale, le metafore e immagini hanno un pregnante senso gnoseologico, non semplicemente illustrativo od ornamentale. L'immagine della barca alla deriva trascinata qua e là dal vento secco, e cioè tempestoso (visto che la tempesta si spiegava come urto fra vapori secchi e umidi), proveniente dalla povertà - comparata cioè con l'elemento terreno, materiale, dal quale scaturisce il vapore - ha anche un forte valore ontologico, se consideriamo che l'idea della navigazione rappresenta la vita umana in quanto portata dallo slancio teleologico delle cose al loro proprio fine naturale «per lo gran mar de l'essere», come spiega Beatrice in Pd. I 109-114 («Ne l'ordine ch'io dico sono accline / tutte nature, per diverse sorti, / più al principio loro e men vicine; / onde si muovono a diversi porti / per lo gran mar de l'essere, e ciascuna / con istinto a lei dato che la porti»). In questo modo, la povertà ferma anche «l'istinto» (v. 114) che dovrebbe portare l'essere umano alla propria auto-realizzazione come natura provveduta e cioè alla propria felicità, e suppone una vera rottura dell'ordine teleologico universale. Vediamo così come la nuova comprensione ed esperienza della povertà si innestano in una cosmovisione ancora precapitalista, in un profondo momento, quindi, di crisi ideologica.

${ }^{25}$ «Pertanto, pianga, pianga la più alta aristocracia toscana, che rifulgeva per un tanto uomo, e piangano tutti i suoi amici e i sudditi, la cui speranza fu crudelmente colpita dalla morte; tra i quali ultimi, anch'io misero devo piangere, io che, cacciato dalla patria ed esule senza colpa, considerando di continuo le mie disgrazie, mi consolavo in lui, con cara speranza. [...] E io, oltre a ciò, in quanto vostro, mi appello alla vostra discrezione per l'assenza alle lacrimose esequie; poiché non sono stato trattenuto dalla negligenza o dalla ingratitudine, ma dalla inattesa povertà dell'esilio. Infatti questa, come una feroce persecutrice, cacciò nella spelonca della sua prigionia me, che mancavo di armi e cavalli, e mentre mi sforzo di levarmi, con tutte le mie forze, lei, fin qui prevalendo, empia, si forza di trattenermi.» (Citiamo dall'edizione Villa, Dante Alighieri, Epistole, op. cit., p. 1436-1439). 
inmeritus», la conseguente povertà che appunto produce l'impossibilità da parte del povero di occupare il suo luogo nel mondo. È importante sottolineare che la povertà si traduce in una perdita delle armi e del cavallo, il che nella concezione puramente feudale della povertà era il sintomo allegorico della perdita da parte del cavaliere del luogo naturale nel mondo, ${ }^{26} \mathrm{e}$ che ora diventa - probabilmente senza perdere del tutto l'antica connotazione sacralizzata - una causa sociale effettiva diretta che, impedendo al povero di mostrare pubblicamente la sua nobiltà o valore individuale e di accedere così a uno stato professionale benestante, gli preclude il suo sviluppo sociale e lo ostacola al momento di presentarsi come uomo-merce, come rappresentazione, nel mercato.

4. È perciò chiave il fatto che in Dante l'esperienza della povertà viene immediatamente vissuta come una crisi di rappresentazione personale nel mondo pubblico, di immagine sociale collegata al proprio ruolo di uomo-merce che deve vendere la sua vita nelle corti italiane, come si vede nella continuazione del passo sopra citato in cui dichiara la sua dolorosa povertà:

La fama buona, principalmente [è] generata dalla buona operazione nella mente dell'amico e da quella è prima partorita; ché la mente del nimico, avegna che riceva lo seme, non concepe. Quella mente che prima la partorisce, sì per far più ornato lo suo presente, sì per la caritade dell'amico che lo riceve, non si tiene alli termini del vero ma passa quelli. $\mathrm{E}$ quando per ornare ciò che dice li passa, contra conscienza parla; quando inganno di caritade li fa passare, non parla contra essa. La seconda mente che ciò riceve, non solamente alla dilatazione della prima sta contenta, ma 'l suo riportamento, sì come qu[as]i suo effetto, procura d'adornare; $\mathrm{e}$ sì, che per questo fare e per lo 'nganno che riceve della caritate in lei generata, quella più ampia fa che a lei non vène, e [con] concordia e con discordia di conscienza come la prima. E questo fa la terza ricevitrice e la quarta, e così in infinito si dilata. E così, volgendo le cagioni sopra dette nelle contrarie, si può vedere la ragione della infamia, che simigliantemente si fa grande. Per che Virgilio dice nel quarto dello Eneida che la Fama vive per essere mobile e acquista grandezza per andare. Apertamente adunque veder può chi vuole che la imagine per sola fama generata sempre è più ampia, quale che essa sia, che non è la cosa imaginata nel vero stato. (Convivio I III 7-11; corsivi nostri)

È quindi molto significativo che l'esperienza della povertà diventi immediatamente crisi di rappresentazione pubblica. Dante capisce - o forse solamente intuisce - che nel nuovo mondo mercantile tutto si gioca nel territorio dell'immagine e quindi la dissociazione fra ambito materiale e ambito della rappresentazione sociale è già una condizione ideologicamente insormontabile. L'immagine o rappresentazione sociale si produce «nella mente dell'amico», o del

${ }^{26}$ «[La povertà] Comporta l'impossibilità di mantenere il suo 'stato' naturale, cioè di acquistare, conservarne o recuperarne gli strumenti o i segni: per esempio, l'aratro o il bestiame per il contadino, l'attrezzo per l'artigiano, la bottega per il mercante, le armi e il cavallo per il nobile, i libri per il chierico, ed anche il pastorale e la mitra per il vescovo». (Mollat, «Il concetto della povertà nel Medioevo: problematica», op. cit., pp. 6-7). 
nemico nel caso dell'infamia, e poco a poco si va staccando dalla veracità materiale, dilatandosi nel bene o nel male e quindi diventando autonoma rispetto alla «buona operazione» originaria che l'ha partorita. Questa immagine, quindi, si carica così di un plusvalore che fa appunto sì che l'individuo si trasformi in merce, cioè in segno o immagine nello spazio semiotico del mercato.

Perciò è importantissimo, per dirla in termini moderni, avere una bella "presenza», proiettare un'immagine di sé che, nella continua concorrenza fra immagine mentale creata dalla fama e immagine presente arrivata attraverso i sensi, non restringa troppo in negativo la proiezione «sociale» della persona e permetta di apprezzare le buone operazioni che costituiscono la sua «bontà». Questa situazione, infatti, è considerata da Dante come il trionfo dell'immagine «di fuori» sulla bontà interiore, e cioè del giudizio «secondo senso» sul giudizio «secondo ragione»:

La maggiore parte delli uomini vivono secondo senso e non secondo ragione, a guisa di pargoli; e questi cotali non conoscono le cose se non semplicemente di fuori, e la loro bontade, la quale a debito fine è ordinata, non veggiono, per ciò che hanno chiusi li occhi della ragione, li quali passano a veder quello. Onde tosto veggiono tutto ciò che ponno, e giudicano secondo la loro veduta. E però che alcuna oppinione fanno nell'altrui fama per udita, dalla quale nella presenza si discorda lo imperfetto giudicio che non secondo ragione ma secondo senso giudica solamente, quasi menzogna reputano ciò che prima udito hanno, e dispregiano la persona prima pregiata. Onde appo costoro, che sono, ohmè, quasi tutti, la presenza ristringe l'una e l'altra qualitade. Questi cotali tosto sono vaghi e tosto sono sazii, spesso sono lieti e spesso tristi di brievi dilettazioni e tristizie, tosto amici e tosto nemici: ogni cosa fanno come pargoli, sanza uso di ragione. [...] Per che manifestamente si vede che per impuritade, sanza la quale non è alcuno, la presenza ristringe lo bene e lo male in ciascuno più che' 'l vero non vuole. Onde, con ciò sia cosa che, come detto è di sopra, io mi sia quasi a tutti l'Italici apresentato, per che fatto mi sono più vile forse che 'l vero non vuole non solamente a quelli alli quali mia fama era già corsa, ma eziandio alli altri, onde le mie cose sanza dubbio meco sono alleviate; conviemmi che con più alto stilo dea [al]la presente opera un poco di gravezza, per la quale paia di maggiore autoritade. (Convivio I Iv 3-5, 12-13; corsivi nostri)

L'aspetto decisivo, a nostro parere, è che l'immagine sociale e il suo correlato negativo, la povertà, implicano di nuovo una rottura ontologica dell'ordine universale, perché l'uomo-merce - come la ricchezza-merce che è distinta dalla cosa-natura $-^{27}$ è

${ }^{27}$ «E prima ch'io ciò dimostri, è da dichiarare un dubio che pare consurgere: che, con ciò sia cosa che l'oro, le margherite e li campi perfettamente forma e atto abbiano in loro essere, non pare vero dicere che siano [cose] imperfette. E però si vuole sapere che, quanto è per esse, in loro considerate, cose perfette sono, e non sono ricchezze, ma oro e margherite [e campi]; ma in quanto sono ordinate alla possessione dell'uomo, sono ricchezze, e per questo modo sono piene d'imperfezione». ( $\mathrm{C} v$. IV XI 5). Come ha spiegato Umberto Carpi: «Le ricchezze in quanto metalli ovvero pietre preziose ecc., osserva Dante, in natura non hanno di per sé imperfezione, non sono cioè ricchezze così come l'oro e 
l'uomo staccato dal «debito fine» al quale è ordinato, cioè rimasto fuori dal movimento teleologico e dall'ordine universali. Il "giudizio secondo senso» che costituisce l'essenza del comportamento mercantile non è in grado di capire le nature in quanto nature e l'uomo in quanto uomo, cioè in quanto essere inseriti in un ordine universale - e quindi non è in grado di apprezzare le opere buone in cui si materializza la nobiltà di animo -, ma li percepisce, non tanto o non solo come una falsa apparenza dissomigliante della verità interiore, ma piuttosto come un qualcosa di alieno a quell'ordine, perso alla deriva nel gran mare dell'essere. Così per Dante l'uomo in stato mercantile altro non è che «legno sanza vela e sanza governo» che ha perso le radici trascendenti che lo conformano come vero uomo.

Si noti che questa configurazione ideologica è la stessa di quella che abbiamo visto in Poscia ch'Amor, in cui i leggiadri cercano il giudizio sensuale del volgo e cercano con ambizione, senza successo, l'accettazione dei buoni, i quali invece giudicano «secondo ragione» «pregiando» non l'abito ma il senno e il cuore gentile. Se in Poscia ch'Amor lo spreco delle ricchezze da parte dei falsi leggiadri, cioè dei «falsi cavalier', malvagi e rei» (v. 112), stimola il giudizio «secondo senso» del volgo e cioè lo staccarsi delle cose e delle persone dall'ordine universale facendole diventare merci, nei primi capitoli del Convivio, scritti, secondo noi, non molti anni dopo, quell'azione disordinatrice - o piuttosto distruttrice o pervertitrice - corrisponde alla povertà, che impedisce che le «opere perfette» - quelle proprie della leggiadria e quindi della nobiltà -, cioè la «buona operazione» da cui dovrebbe scattare la fama - come abbiamo visto sopra in Convivio I III 7 - siano apprezzate dalla voce pubblica e considerate come «a debito fine ordinate». Il problema quindi, sia per eccesso sia per difetto, consiste nell'introdurre il giudizio nel falso mondo delle cose e degli uomini diventati merci, o ricchezze, considerati quindi solamente in funzione del loro «valore» sociale-mercantile e non del loro «valore» nell'insieme del cosmo, dell'ordine bello universale. ${ }^{28}$ Secondo i vv. 102-106 della canzone («cotante / persone quante / sembiante portan d'omo, e non risponde / il lor frutto alle fronde / per lo mal c'hanno in uso»), nel leggiadro una fronda abbondante e vistosa nasconde la mancanza di frutto, cioè la mancanza di buone azioni od operazioni, dilettevoli e proporzionate al soggetto che le compie e aggiustate razionalmente a un

l'argento nel ventre della terra non costituiscono in sé tesoro ('l'oro e l'argento non sono per natura denaro, ma il denaro è per natura oro e argento' , sintetizzerà sei secoli dopo Marx) [...]. Per divenire imperfetti, per comportare cioè quella tendenza alla dismisura nel raunare e nello spendere che costituisce il comportamento opposto alla nobile larghezza (alla prudenza e alla giustizia) [...] è necessario che i metalli si trasformino in moneta, le pietre preziose e i campi in beni misurabili dal denaro: cioè la natura in valore mobile, donde cupidigia e prodigalità». (Carpi, U., La nobiltà di Dante, Firenze, Polistampa, 2004, vol. I, pp. 63-64)

${ }^{28}$ Una conseguenza di questa concezione è che le merci e gli uomini-merce sono per Dante sempre brutti, perché la bellezza solo si acquisisce all'interno dell'ordine universale come conseguenza della funzione, o valore, entro quest'ordine. Se la vera leggiadria è essenzialmente bella, appunto perché in essa il valore e l'apparenza hanno una corrispondenza perfetta, la bellezza non è possibile nel disordine mercantile. 
debito fine: ${ }^{29}$ invece nel povero una fronda venuta meno, scarsa e poco vistosa, fa sì che i suoi frutti, le sue azioni o prodotti, non vengano considerati e goduti dagli altri, sommettendolo così a un vero e proprio processo di mercificazione ovvero di s-fruttamento. $^{30}$

Vediamo in questo modo come tutta l'ideologia sacralizzata delle apparenze terrene, delle divergenze abissali fra senso e ragione, cioè fra corpo e anima o fra mondo sublunare e mondo supralunare, con le quali Dante è alle prese durante tutta la sua vita intellettuale mediante soprattutto la questione dell'amore - o, se si vuole, con l'amore come strumento ideologico per cercar di disinnescarla - si innesta in una vicenda di tipo psicosociale che non è più feudale ma prettamente capitalista, cosicché la dissomiglianza sensuale del mondo terreno deve servire a spiegare, a riconciliare, la terribile e oscura esperienza personale di essere diventato uomo-merce e di dover quindi necessariamente ornarsi per mettersi in vendita nel mercato tutto sensuale dei non saggi. La cosa terribile è che, malgrado la cupa consapevolezza che Dante mostra di questa situazione, non può far altro che arrendersi ad essa, impostando la sua opera filosofica come un «biglietto da visita», per dirla crudamente, che gli restituisse un'immagine di autorità con valore economico professionale ${ }^{31}$ («conviemmi che con più

${ }^{29}$ Spiega Tommaso d'Aquino che il termine frutto indica sempre le azioni umane se sono convenienti al soggetto che le compie e seguono la ragione: «Si autem dicatur fructus hominis id quod ex homine producitur, sic ipsi actus humani fructus dicuntur, operatio enim est actus secundus operantis, et delectationem habet, si sit conveniens operanti. Si igitur operatio hominis procedat $\mathrm{ab}$ homine secundum facultatem suae rationis, sic dicitur esse fructus rationis. Si vero procedat ab homine secundum altiorem virtutem, quae est virtus spiritus sancti; sic dicitur esse operatio hominis fructus spiritus sancti». (Tommaso d'Aquino, Somma di teologia I-II q. 70 a. 1 co.; citiamo da Tommaso d'Aquino, La somma teologica: sola traduzione italiana, a cura della redazione delle ESD, Bologna, PDUL Edizioni, 1996, reperibile al sito telematico http://www.carimo.it/sommateologica/somma.htm).

${ }^{30}$ La metafora agricola del frutto e le fronde compare diverse volte nell'opera di Dante: ad esempio in tutta la spiegazione sulla nobiltà e i suoi risultati in Convivio IV XXI, o nell'offerta di Carlo Martello a Dante in Paradiso VIII 55-58, o nel discorso di Beatrice contro l'umana cupidigia in Paradiso XXVII 124-126, 148, ecc.

${ }^{31}$ Sulle strategie di «auto-autorizzazione» di Dante nel Convivio, iniziando dalla pratica dell'autocommento, ha scritto pagine imprescindibili Albert Russell Ascoli (Ascoli, A.R., Dante and the Makind of a Modern Author, Cambridge, Cambridge University Press, 2008), che parte dalla «decisione di Dante di considerare le proprie canzoni volgari degne di commento, collocandole cosí, implicitamente, nella posizione formale di auctoritates e ponendo se stesso in quella di auctor» (citiamo dalla traduzione italiana di Anna Montanari, reperibile su academia.edu, p. 70). Da parte sua, Sabrina Ferrara, nel libro già citato (Ferrara, La parola dell'esilio, op. cit.) ripercorre brillantemente la successione di strategie di autorappresentazione da parte di Dante durante l'esilio e il rapporto che esse implicano coi lettori (di cui tratta anche Ascoli; e Zanin, E., «Dante's Implied Reader in the Convivio», in F. Meier (a c. di), Dante's Convivio. Or How to Restart a Career in Exile, Oxford-Bern-Berlin-Bruxelles-New York-Wien, Peter Lang, 2018, pp. 207-221; per chi Dante cerca «an audience which might acknowledge his authority [...] which may agree with his notion of wisdom and nobility and therefore recognize Dante as a true noble and ultimately a wise man», p. 
alto stilo dea [al]la presente opera un poco di gravezza, per la quale paia di maggiore autoritade»). ${ }^{32}$ In questo modo Dante si vede immerso in un mondo che coscientemente rifiuta ma che inconsciamente deve assumere e cercar di spiegare con i propri strumenti intellettuali, ancora non adatti a quella nuova situazione psico-sociale. Solo quando riuscirà a superare intellettualmente, e direi anche vitalmente, la sua condizione di uomo-merce, sarà in grado di ritrovare la sua rotta personale per il gran mare dell'essere.

Juan Varela-Portas de Orduña jivarelaportas@filol.ucm.es

Fecha de recepción: 11/09/2019

Fecha de aceptación: 03/02/2020

220). Ferrara differenzia la «autocostruzione» come filosofo e studioso (auctoritas) del Convivio e il De vulgari eloquentia, come politico delle Epistole, e finalmente come profeta (la quale include le due prime) nella Commedia: «Poiché è ancora l'esilio che determina il bisogno di ricostruire e di proporre un'immagine di sé al lettore modificando la realtà storica, vissuta come ingiusta, il senso del testo non può essere dissociato né dalle contingenze reali che lo hanno generato né da colui che lo ha concepito». (p. 19)

${ }^{32}$ In questo senso, si deve ammettere che Dante cade in contraddizione perché si avvicina a quelli che fanno della «letteratura» una meretrice, cioè che usano il sapere in vista del guadagno di denaro o dignità (cioè prestigio o capitale sociale), criticati aspramente nel celebre capitolo nono del primo trattato del Convivio (e anche in III XI 10). Certo che la finalità di proiettare un'immagine socio-mercantile di gravità e auctoritas è secondaria e influisce soprattutto nello stile - che si considera ancora come tecnica retorica e non come proiezione della personalità o anima dell'autore, come sarà da Petrarca in poi -, cioè sulla forma o bellezza o parte persuasiva del discorso e non sul contenuto o bontà, ma si deve comunque convenire che Dante qua si avvicina alle posizioni utilitaristiche degli intellettuali del Comune, imposte dalla nuova società mercantile e alle quali Dante, anche se i suoi «clienti» saranno principalmente i signori delle corti feudali, non potrà più sottrarsi. 